\section{Phonological and semantic representations of words, compartments of memory, and rehearsal*}

\author{
RICHARD M. WEIST and CHARLOTTE CRAWFORD \\ University of Nebraska-Lincoln, Lincoln, Nebr. 68508
}

The effect of inhibiting rehearsal on Ss' capacity to remember phonological and semantic representations was studied with bilingual and nonbilingual Ss in a semifree recall task. The procedure used to retard rehearsal caused an overall decrement in the capacity of bilingual and nonbilingual Ss to remember words in both Cantonese and English. Inhibiting rehearsal did not cause a relatively greater decrement in the nonbilinguals' recall of foreign language words.

Waugh \& Norman (1965) initiated the idea that rehearsal involves an exchange of information between working memory, i.e., PM or STS, and permanent memory, i.e., SM or LTS. Atkinson \& Shiffrin (1968) developed this idea further in their tricompartmental model. Rehearsal was viewed as a repetition or recopying of working memory representations. The function of rehearsal was to maintain "information ensembles" in working memory. Working memory regeneration resulted in the increase in the strength of some corresponding permanent memory trace. Rundus (1971) pointed out that the exchange of information proposed by Waugh and Norman and by Atkinson and Shiffrin was unidirectional, i.e., from working to permanent memory. In experiments which monitored the contents of a rehearsal buffer (e.g., Rundus, 1971; Weist, in press), the unidirectional claim was found to be counterfactual. The content of a rehearsal buffer depends on information retrieved from permanent memory as well as the presentation sequence.

A meaningful discussion of rehearsal processes is predicated on the understanding of the concept of a memory representation. Without claiming an adequate semantic theory, Kintsch (1970) provides a useful framework for conceptualizing the encoding and recoding of words. The permanent memory representation of a word, i.e., the lexical concept, was viewed as a set of distinctive features or markers and a rule system relating the features. Three feature subsets or vectors were distinguished: phonemic (P), image (I), and syntactic-semantic (SS) features. Synthesizing the

*This research was supported by a University of Nebraska-Lincoln Research Council grant. No. W42-4128-28A. We would like to thank Lam Chock Hing, who helped prepare the tapes. theoretical arguments of Kintsch (1970) and Neisser (1967) with research on the effects of encoding context (e.g., Thomson \& Tulving, 1970 ), Weist (in press) proposed that rehearsal involved a context-sensitive act of reconstruction which provides the occasion and the mechanism for changing, as well as maintaining working memory feature sets, i.e., information ensembles. This interaction between working and permanent memory was viewed as changing the familiarity value of lexical concepts as well as revising the contents of working memory (see Kintsch, 1970, on "cognitive work").

The current research was designed to explore the effects of rehearsal on remembering when there is a clear possibility for interaction between working memory and permanent memory and when the memory representation of a word is relatively limited to a phonological representation in working memory and relatively isolated from the semantic features of a lexical concept. Bilingual and nonbilingual $\mathrm{Ss}$, mixed-language lists, and an addition procedure to retard rehearsal were employed to study this problem. The bilingual person has been found to utilize SS and $P$ features in both working and permanent memory to remember a mixed-language list of words. The nonbilingual person is relatively limited to maintaining $P$ features of foreign words in working memory. The term "relative" is used in this context because it is possible for Ss to generate SS features to mediate the retention of otherwise meaningless words. Posner \& Rossman (1965) and others have found that limiting Ss' capacity to rehearse decreases retention. For the bilingual person, restricting rehearsal limits the interaction between compartments of memory and thus the revision of information ensembles and the familiarity values of associated lexical concepts. In the nonbilingual person, restricting rehearsal limits the number of phonological representations that can be maintained in working memory. Because of the phonological nature of the representation, and the fact that remembering is relatively isolated from permanent memory, it was expected that nonbilingual Ss would be able to remember relatively few foreign words when rehearsal was restricted.

The Ss were 40 introductory psychology students at the University of Nebraska-Lincoln. Twenty of the Ss were from Hong Kong and were fluent in Cantonese and English. The other 20 Ss were all native English speakers, and none spoke any Chinese language. Chinese and English Ss signed up separately to participate. The experimental treatments were randomly alternated, with Ss being assigned to a treatment when they appeared for the experiment. Two stimulus lists were made up of 12 Cantonese words and 12 different English words. Eight semirandom presentations of each list were prepared on tape, with the English words spoken by a native English speaker and the Cantonese words spoken by a Cantonese-English bilingual having Cantonese as his native language. The order of presentation was restricted so that an equal number of Cantonese and English words occurred in each fourth of the list. The words were presented at a 5 -sec rate. On one list (add list), after each word, the English speaker presented a single-digit number from 1 to 5 .

The instructions were presented in English for the nonbilingual Ss and in both languages for the bilingual Ss. The Ss were told that the list was made up of 12 English and 12 Cantonese words, that they were to recall the words by alternating one English word and one Cantonese word in any order, and that the pairs could be recalled in any order. These instructions were used so that Ss would try to remember words from both languages. The Ss were told that when they had recalled as many of the words as they could remember by alternating languages, they were to recall as many other words as they could remember in either language and in any order. These instructions allow for a possible Cantonese-English imbalance in recall. Ten Ss from each language group were given the lists having word-number pairs and were told to give the sum of the numbers before recalling the words. The Ss were told that their totals would be checked and were given feedback concerning their totals at the end of the output phase. If incorrect, they 

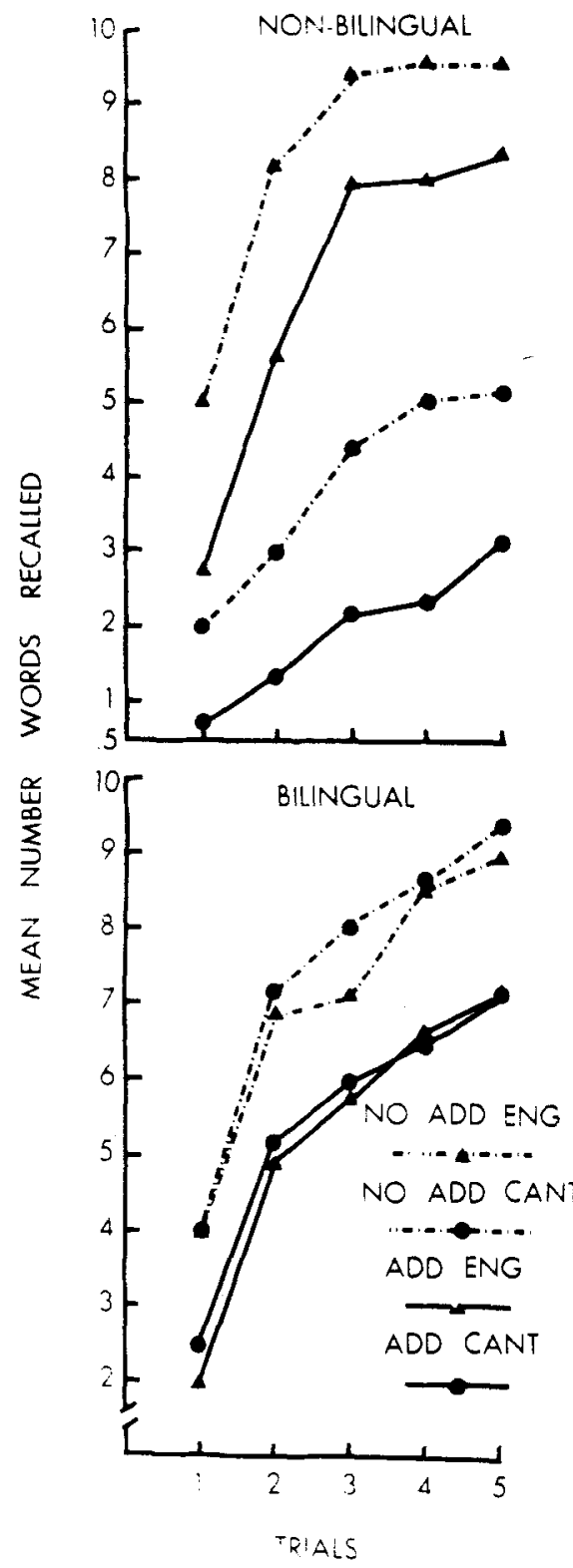

Fig. 1. Bilingual and nonbilingual recall of English (ENG) and Cantonese (CANT) words under the addition procedure (ADD) and under the semifree recall procedure (NO-ADD).

were encouraged to try harder. These precautions were needed for $S s$ to attend to the addition task consistently. The stimulus words were presented to all Ss at a 5 -sec rate with a Sony 500 tape recorder, and the responses were recorded on a Panasonic tape recorder. All Ss received eight semifree recall trials.

There were two types of Ss [bilingual (BI) and nonbilingual (NON-BI)], two kinds of lists and instructions [addition (ADD) and nonaddition (NO ADD)], $10 \mathrm{Ss}$ in each of the four groups, two languages (Cantonese and English), and eight trials. The data were evaluated with an analysis of variance having two factors between-Ss and two within-Ss.

RESULTS AND DISCUSSION

Figure 1 shows that the addition procedure caused a decrement in recall and that the Cantonese words were harder for the English Ss to remember. These observations were reflected in the analysis of variance, which indicated that the following main effects were significant: $S$ type, $\mathrm{F}(1,36)=10.4, \mathrm{p}<.005 ;$ instructions, $\mathrm{F}(1,36)=30.0, \mathrm{p}<.001 ;$ language $F(1,36)=96.5, p<.001$; and trials, $\mathrm{F}(4,144)=150.0, \mathrm{p}<.001$. The interactions of $\mathrm{S}$ Type by Language $[F(1,36)=122.0, \quad p<.001]$, Language by Trials $[\mathrm{F}(4,144)=\mathbf{5 . 5 0}$, $\mathrm{p}<.001]$, and $\mathrm{S}$ Type by Language by Trials $[F(4,144)=5.57, p<.001]$ were also significant. None of the other interactions were significant $(p>.10)$. The fact that the addition procedure did not interact with $S$ type and language indicated that inhibiting rehearsal did not cause a relatively greater decrement on information represented primarily in working memory on a phonological dimension.

There has been considerable support for the proposition that the recency effect of a serial position curve represents the retrieval of information from working memory (e.g., Glanzer \& Cunitz, 1966; Kintsch \& Buschke, 1969; Waugh \& Norman, 1965). If the contents of a rehearsal buffer is dependent on the order of input such that old items are replaced by new items, then items in final input positions will be highly accessible at the time of recall. According to Atkinson \& Shiffrin (1968), Ss select items from the input for inclusion in a rehearsal buffer. The longer an item is maintained in the rehearsal buffer, i.e., the more the item is rehearsed, the greater the buildup of strength of that item in permanent memory. If new items replace old items in the buffer or if replacement is random, items in final positions have a higher probability of being available in the buffer at the time of recall. Thus, the recency effect is attributed to retrieval of immediately accessible items from the buffer, and the primacy effect is attributed to the greater strength of items in the initial positions. Applying this argument to the present experiment, the serial position curves for the nonbilinguals' recall of foreign words should show a recency effect and no primacy effect, but the results of this experiment show just the opposite to be the case.

In preparing the list, four English words and four Cantonese words were assigned randomly to the first, second, and third eight positions. The position analysis was based on the proportion of English words and the proportion of Cantonese words correctly recalled in the initial, middle, and final eight positions. Table 1 shows the proportion correct as a function of $S$ type, instructions, and position. These data were summed over trials, and the position curves are flatter than on any individual trial; however, the trends are representative. When Ss are free to rehearse, there is a primacy and recency effect for bilingual Ss on both languages and for nonbilingual Ss on English words. The nonbilingual recall of foreign words was characterized only by a primacy effect. The imposition of the addition procedure tends to flatten all of the position curves. A position analysis was computed for the four groups separately. The position effects were significant in NO ADD conditions but not in ADD conditions; NO-ADD/NON-BI, $F(2,18)=15.5$, $\mathrm{p}<.001 ; \quad \mathrm{NO}-\mathrm{ADD} / \mathrm{BI}, \quad \mathrm{F}(2,18)=$ $8.06, \mathrm{p}<.01, \mathrm{ADD} / \mathrm{NON}-\mathrm{BI}, \mathrm{F}<1$; ADD $/ \mathrm{BI}, \mathrm{F}(2,18)=2.28, \mathrm{p}>.10$. The interaction of Position by Language was never significant. The design was not sensitive to the presence of a recency effect in the nonbilinguals' recall of English words and the absence of a recency effect when recalling Cantonese words, $F(2,18)=$ $1.23, \mathrm{p}>.10$.

Two alternative hypotheses were evaluated regarding the primacy effect in the nonbilingual Ss' recall of foreign words. Nonbilingual Ss may have rehearsed old or initial-position

Table 1

Mean Proportion of Cantonese and English Words Correctly Recalled in the Initial, Middle, and Final Positions

\begin{tabular}{llllccc}
\hline & & & \multicolumn{3}{c}{ Position } \\
\cline { 5 - 6 } & & & $1-8$ & $9-16$ & $17-24$ \\
\hline \multirow{3}{*}{ Nonbilingual } & Cantonese & No Add & .40 & .30 & .26 \\
& & Add & .18 & .16 & .15 \\
& English & No Add & .76 & .62 & .68 \\
& & Add & .55 & .53 & .53 \\
& Cantonese & No Add & .62 & .56 & .63 \\
& & Add & .45 & .40 & .48 \\
& English & No Add & .64 & .52 & .58 \\
& & Add & .51 & .38 & .39 \\
\hline
\end{tabular}


Cantonese words and rejected the new Cantonese words from the rehearsal buffer. According to this hypothesis, it might be expected that the first words in the presentation would be the first words in recall. In order to evaluate this hypothesis, the likelihood of early recall of initial position and final position Cantonese and English words was determined. A word in the final eight positions was defined as having been recalled from a rehearsal buffer if the word was recalled within a span of seven words from presentation to recall (see Waugh \& Norman, 1965). A similar analysis was performed on the initial eight words. For the words in the initial eight positions, the proportion of words meeting the early recall criterion was .239 for Cantonese and .308 for English. For the words in the final eight positions, these proportions were .549 for Cantonese and .457 for English. This analysis does not support the hypothesis that the initial four Cantonese words were more likely to be recalled from a buffer. A second alternative hypothesis was suggested by the data but could not be adequately tested. The overall probability of recalling each Cantonese word was determined. Words that sound to the nonbilingual like "hi" and "mow" were easier to remember than words like "sokfu" and "moak." It is possible that the easier items were recoded utilizing mnemonic aids. This kind of processing may have been more effective for words in the initial positions.

In general, these findings support the view that rehearsal involves an interaction between working memory and permanent memory. When this interaction is restricted, recall decreases and serial position curves are flattened. When this interaction is severely retarded by presenting words which have no lexical concepts associated with the words, recall is also inhibited. In this experiment, these two factors did not interact. The rehearsal of foreign words as well as the rehearsal of native words may involve an interaction between working and permanent memory. In the case of native words, the features of associated lexical concepts are involved, and in the case of foreign words, semantic features are recruited as memory aids.
REFERENCES

ATKINSON, R. C., \& SHIFFRIN, R. M. Human memory: A proposed system and its control processes. In K. W. Spence and J. T. Spence (Eds.), The psychology of learning and motivation: Advances in research and theory. Vol. II. New York: Academic Press, 1968, Pp. 90-195.

GLANZER, M. \& CUNITZ, A. R. Two storage mechanisms in free recall. Joumal of Verbal Learning \& Verbal Behavior. $1966,5,351-360$.

kINTSCH W. Models for free recall and recognition. In D. A. Norman (Ed.), Hodels of human memory. New York: Academic Press, 1970. Pp. 331-373.

KINTSCH. W. \& BUSCHKE, H. Homophones and synonyms in short-term memory. Journal of Experimental Psychology, 1969,80, 403-407.

NEISSER, U. Cognitive psychology. New York: Appleton-Century-Crofts, 1967.

POSNER, M. I \& ROSSMAN, E. Effects of size and location of informational transforms upon short-term retention. Journal of Experimental Psychology, 1965, 70, 496-505.

RUNDUS, $\dot{D}$. Analysis of rehearsal processes in free recall. Journal of Experimental Psychology, 1971, 89, 63-77.

THOMSON, D. M., \& TULVING, E. Associative encoding and retrieval: Weak and strong cues. Journal of Experimental Psychology, 1970, 88, 255-262.

WAUGH, N. C.. \& NORMAN, D. A. Primary memory. Psychological Review, 1965, 72, 89-104.

WEIST. R. M. The role of rehearsal: Recopy or reconstruct. Journal of Verbal Learning \& Verbal Behavior, in press. 